Stress, Burnout, and Psychosocial Support for Staff of the Emergency Medical Services

Jana Seblova; ${ }^{1}$ Dana Hlavackova; ${ }^{1}$ Vladimír Kebza, ${ }^{2}$

Jana Vignerova; ${ }^{2}$ Blanka Cepicka ${ }^{3}$

1. Ministry of Health of the Czech Republic-EMS

2. National Institute of Public Health; 3SENA, Prague, Czech Republic

Introduction: The objectives of study on stress of EMS professionals were to analyze the average and individual rates of burnout syndrome symptoms, sources of stress associated with the profession, and the degree of exposition to critical incidents. Two years later, burnout and experience with any psychological intervention technique were analyzed again. Meanwhile, Critical Incident Stress Management (CISM) techniques after critical incidents, according to Mitchell's model, were performed in some organizations' lectures on prevention, communication trainings, consultations concerning professional stress, supervision meetings and debriefings also are offered to professionals within crisis preparedness.

Methods: The questionnaire study used a screening test of symptoms of burnout syndrome. Burnout scores of groups from the year 2003 (286 respondents) and from 2005 (597) were compared. Analysis of variance (ANOVA) was used for basic comparison. The General Linear Model (GLM) was used for testing individual factors' influence on burnout symptoms and for comparison for each of four professional groups: physicians, medical nurses, paramedics, and dispatchers. Exposition to critical incidents, and stressful and positive factors associated with profession were analyzed using descriptive statistics.

Results: A highly significant difference in burnout score was found between the group of dispatchers when compared to any other professional group. The other factor significantly influencing symptoms of burnout was the length of practice in EMS. No other factor (sex, matrimonial status, any professional position other than dispatcher, age) has had influence on the degree of burnout. Surprisingly, high (85.5\%) experience with assault and/or an ambulance vehicle accident during duty was found. Experience with any psychological intervention technique was also analyzed. No statistically significant difference $(p=0.771)$ was found between the two groups: A (372; any type of intervention) and $B(225$; no intervention). Satisfaction with the named techniques ranged from 79-92\%. Another pilot study with psychological peer support took place in Prague in 2008. This pilot testing identified the most stressful events and the need of support (15 contacts/month, on average). Effecivness of both models-CISM and peercurrently is being evaluated

Conclusions: A large-scale study on stress of EMS professionals had proved the need for psychological support. Analysis of stress helped to implement supporting psychological techniques adapted to specific needs of rescuers. Based on the results and on experience from pilot programs the Ministry of Health has constituted a working group to develop national guidelines for psychological crisis intervention for rescuers. The Ministry's draft of the Law about Emergency Medical Services also declared the obligation to provide psychological support both for rescuers and victims of disasters. The aim of psychological interventions and targeted education is to increase well-being of the professionals, their effectiveness, and, as a result, to improve the patient care.

Keywords: burnout; critical incidents; emergency medical services (EMS); stress; psychological support Prehosp Disast Med 2010;25(5):s104

\section{Psychological Support for EMS Professionals in the Czech Republic}

Jana Seblova; ${ }^{1}$ Dana Hlavackova; ${ }^{2}$ Blanka Cepicka ${ }^{3}$

1. Ministry of Health of the Czech Republic-EMS

2. Ministry of Health of the Czech Republic

3. S.E.N.A. Prague

Introduction: A study on stress of emergency medical services (EMS) professionals proved the need for psychological support. Based on results of the study and on experience from pilot programs, the Ministry constituted a working group to prepare national guidelines. The Ministry's draft of the Law about EMS also declared the obligation to provide psychological support both for rescuers and victims.

Methods: Prevention, education, supervision meetings and debriefings after critical incidents according to Mitchell's model have been performed in Central Bohemia and Northern Moravia since 2004. A pilot study with crisis intervention by peers occurred in Prague in 2008. Effectiveness of both models currently are evaluated

Results: This study on stress analyzed experiences with any psychological intervention technique. No statistically significant difference $(p=0.771)$ was found between the two groups: A (372; with any intervention) and B (225; with no intervention). Satisfaction with different techniques ranged from $79-92 \%$. Pilot testing of psychological peer support in the Prague's EMS identified the most stressful events and also the need of support. Based on these preliminary results, the working group of the Ministry started to develop national guidelines for psychological crisis intervention for rescuers.

Conclusions: The aim of psychological interventions and targeted education is to increase well-being of the professionals, their effectiveness and, as a result, improvements in the patient care.

Keywords: critical incidents; emergency medical services (EMS); psychological support Prebosp Disast Med 2010;25(5):s104

\section{Human Factors and Medicine Panel Activities in} Psychological Health and Mild TBI/Concussion

Jobn Frazier Glenn

NATO Human Factors and Medicine Panel

Introduction: As in previous eras, military members and civilians involved in current operations are reporting increased incidence of psychological and mental health problems during and after exposure to the physical and mental stresses that are part of armed conflict. The blast effects of Improvised Explosive Devices (IEDs) also have been associated with an increased incidence of mild traumatic brain injury (mTBI), or post-concussive disorder. The causal factors, incidence rates, and options 
for diagnosis, prevention, and treatment are a target of research in many NATO countries.

Methods: This presentation describes recent HFM Panel technical activities related to psychological $/$ mental health and $\mathrm{mTBI}$ and summarizes some of the main findings from those activities.

Results: In preparation.

Conclusions: NATO HFM Panel Technical Activities provide an opportunity for other NATO organizations to benefit from the efforts conducted with the support of the Research and Technology Agency of the Research and Technology Organization.

Keywords: armed conslict; blast effects; mild traumatic brain injury Prebosp Disast Med 2010;25(5)::104-s105

\section{Psychological Team Organization following a Disaster Joana Faria; Márcio Pereira; Jacinta Gonf̧alves; Gabriela Salazar; Sara Rosado; Sónia Cunba; Verónica Oliveira \\ National Institute of Medical Emergency}

Introduction: Following an abnormally stressful event, such as a disaster, people can experience a wide range of psychological and physiological acute reactions, which can be extended to first responders. Psychological first aid for disaster-induced stress and trauma may be a precious resource to facilitate a resilience response. Given the disaster's dimension, the technicians may have to intervene in different places with different objectives.

Methods: This study results from documented research based on the past experience and on the best practices describes in the literature. A search was completed using terms "disaster", "prehospital", and "psychological intervention" using the EBSCO database, MEDLINE and PSYCHARTICLES.

Results: The Centre for Psychological Support and Crises Intervention (CAPIC) of the National Institute of Medical Emergency created a description of six role tasks that can be assumed in a disaster scenario: (1) Psychosocial Coordinator; (2) primary triage; (3) intervention with people in need of medical assistance and intervention with people in no need of medical assistance; (4) support in the area of bodies recognition; (5) intervention with first responders; and (6) support for relatives through the Information Centers. In addition, a triage protocol was produced.

Conclusions: This study suggests that following the proposed role, tasks in a disaster scenario that is, by nature, chaotic, may enhance the psychological intervention, producing a more resilient behavior.

Keywords: disaster; prehospital; psychological intervention; team organization

Prehosp Disast Med 2010;25(5):s105

Psychosocial Resilience as a Cross-Cultural Concept: Understanding and Measuring Psychosocial Resilience Joana Faria

1. National Institute of Medical Emergency

2. Faculty of Psychology and Educational Sciences, Lisbon University

Introduction: Resilience refers to the process of coping with stressors, adversity, change, or opportunity in a man- ner that results in the identification, fortification, and enrichment of protective factors (Richardson, 2002). Resilience has proved to be quantifiable by scales such as the Connor-Davidson Resilience Scale (CD-RISC) (Vaishnavi, Connor, Davidson, 2007). This study examines the psychometric properties of the Portuguese version of the CD-RISC.

Methods: The Portuguese version of the ConnorDavidson Resilience Scale was used. The CD-RISC items were translated with a process of translation and backtranslation by persons fluent in both Portuguese and English. The participants were Portuguese adults from the general population, and of both sexes. The Scale consists of 25 items with an alpha value of 0.89 and test-re-test correlation of 0.87 in the studies with American participants.

Results: Preliminary results of the adaptation study will be presented, including factor structure and psychometric properties of the Portuguese version of the CD-RISC.

Conclusions: It is concluded that the construct of resilience and its measurement used on the American population can be helpful and applicable in understanding Portuguese people ability to thrive despite adversity. Keywords: Connor-Davidson's Resilience Scale; cross-cultural; factor structure; protective factors; resilience; psychometric properties; trauma Prebosp Disast Med 2010;25(5):s105

\section{Trauma in Deployment}

Comparison of Four Hemostatic Agents versus Standard Gauze Dressing in Control of Extremity Hemorrhage in a Model of Penetrating Combat Trauma

\section{LCDR Lanny F. Littlejobn}

Naval Medical Center Portsmouth

Introduction: A randomized, prospective, unblinded trial was conducted to investigate hemostatic agents in a model of severe vascular injury in a small, linear tract wound designed to replicate a penetrating combat injury.

Methods: A complex groin injury with transection of the femoral vessels through a $3 \mathrm{~cm}$ entrance wound and $45 \mathrm{sec}-$ onds of uncontrolled hemorrhage was created in 80 swine prior to randomization to five groups. The groups included: (1) standard gauze (SD); (2) Celox (CX); (3) Chitoflex (CF); (4) Combat Gauze (CG); and (5) WoundStat (WS). Each agent was applied with five minutes of manual pressure prior to resuscitation. Hemodynamic parameters were recorded $>180$ minutes. Primary endpoints included incidence and the amount of rebleeding.

Results: Composite adverse events consisting of mortality, post-treatment hemorrhage, and failure of initial hemostasis were compared between treatment groups using a single degree of freedom chi-squares. Chi-squares were Yates corrected to obtain conservative tests of statistical significance. Four of $16(25 \%) \mathrm{CX}, 10$ of $16(62.5 \%) \mathrm{CF}, 6$ of $16(37.5 \%)$ CG, 11 of $16(68.8 \%) \mathrm{WS}$, and 7 of $16(43.8 \%)$ SD subjects suffered from adverse events. A significant difference was found between the agents CELOX-A and WoundStat with respect to composite adverse events $(p=0.0335)$. 